UCRL-TR-200273

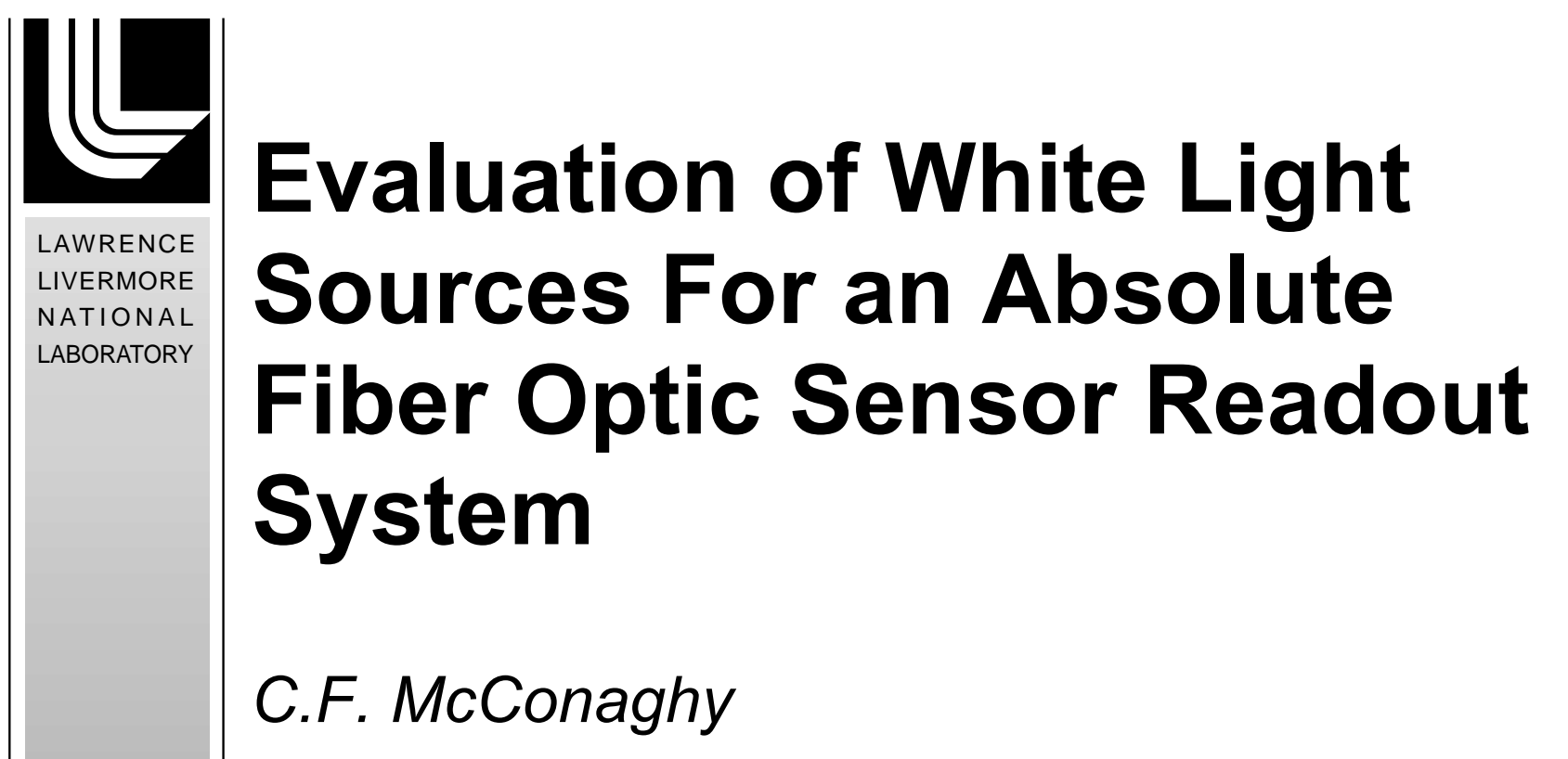

October 10, 2003 
This document was prepared as an account of work sponsored by an agency of the United States Government. Neither the United States Government nor the University of California nor any of their employees, makes any warranty, express or implied, or assumes any legal liability or responsibility for the accuracy, completeness, or usefulness of any information, apparatus, product, or process disclosed, or represents that its use would not infringe privately owned rights. Reference herein to any specific commercial product, process, or service by trade name, trademark, manufacturer, or otherwise, does not necessarily constitute or imply its endorsement, recommendation, or favoring by the United States Government or the University of California. The views and opinions of authors expressed herein do not necessarily state or reflect those of the United States Government or the University of California, and shall not be used for advertising or product endorsement purposes.

This work was performed under the auspices of the U.S. Department of Energy by University of California, Lawrence Livermore National Laboratory under Contract W-7405-Eng-48. 


\author{
Evaluation of White Light Sources \\ For an Absolute Fiber Optic Sensor Readout System \\ C. McConaghy
}

\title{
Introduction
}

This report summarizes work done in pursuit of an absolute readout system for FabryPerot optics sensors such as those built both by FISO and LLNL. The use of white light results in a short coherence length reducing the ambiguity of the Fabry-Perot gap measurement which is required to readout the sensor. The light source coherence length is the critical parameter in determining the ability to build a relative or an absolute system. Optical sources such as lasers and LEDs are rather narrow in optical spectral bandwidth and have long coherence length. Thus, when used in interferometric sensor measurements, one fringe looks much like another and it is difficult to make an absolute measurement. In contrast, white light sources are much broader in spectral bandwidth and have very short coherence lengths making interferometry possible only over the coherence length, which can be 1 or 2 microns. The small number of fringes in the interferogram make it easier to calculate the centroid and to unambiguously determine the sensor gap. This is depicted in the following figure.
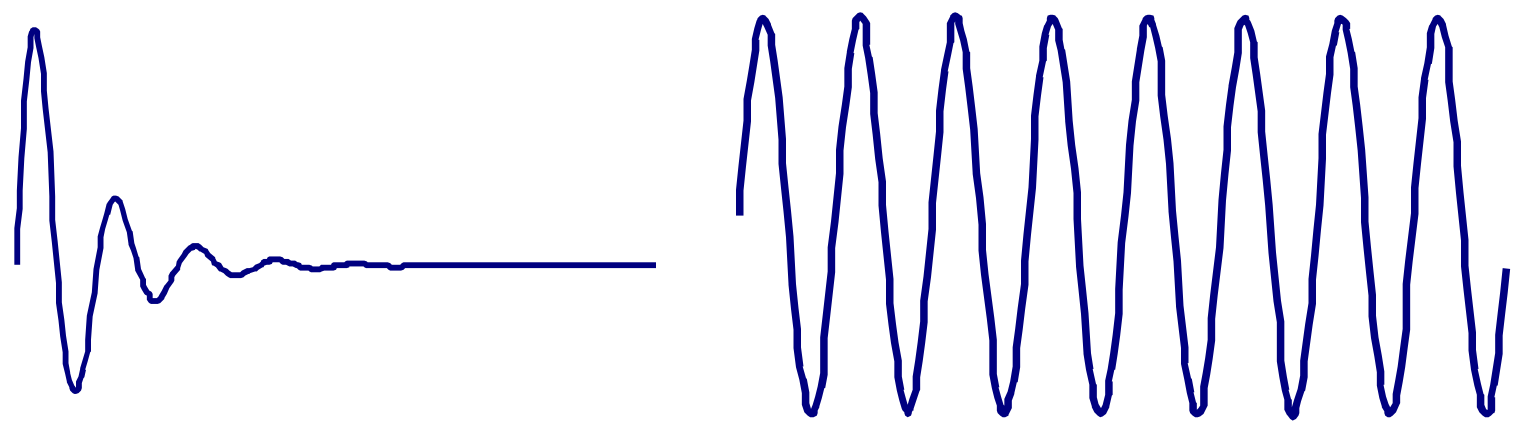

Figure 1: Interference Pattern for a short coherence length white light source (left) and a long coherence length light source such as a Laser. The X-axis is the sensor gap.

However, unlike LEDs and Lasers, white light sources have very low optical power when coupled into optical fibers. Although, the overall light output of a white light source can be hundreds of milliwatts to watts, it is difficult to couple more than microwatts into a 50-micron core optical fiber. In addition, white light sources have a large amount of optical power in spectrum that is not necessarily useful in terms of sensor measurements. The reflectivity of a quarter wave of Titanium Oxide is depicted in Figure 2. This coating of Titanium Oxide is used in the fabrication of the sensor. This figure shows that any light emitted at wavelengths shorter than $600 \mathrm{~nm}$ is not too useful for the readout system. A white light LED spectrum is depicted in Figure 3 and shows much of the spectrum below $600 \mathrm{~nm}$. In addition Silicon photodiodes are usually used in the readout system limiting the longest wavelength to about $1100 \mathrm{~nm}$. Tungsten filament sources may have much of their optical power at wavelengths longer than $1100 \mathrm{~nm}$, which is outside the wavelength range of interest. An incandescent spectrum from a tungsten filament is 
depicted in Figure 4. None of this is to say that other types of readout systems couldn't be built with IR detectors and broadband coatings for the sensors. However, without reengineering the sensors, the wavelength restrictions must be tolerated.

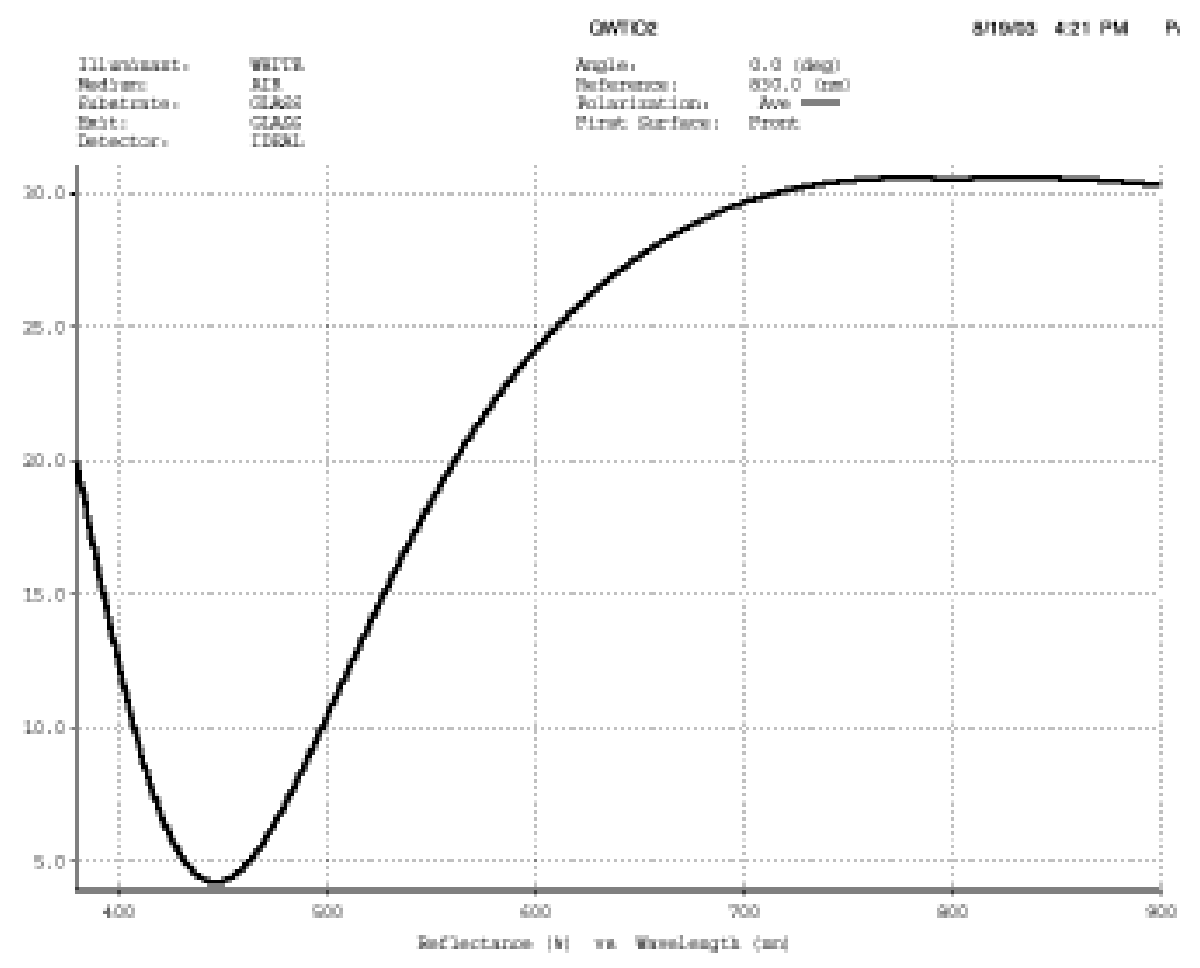

Figure 2: Optical reflectivity of a quarter wave thickness $\mathrm{TiO} 2$ coating. $\mathrm{Y}$-axis is reflectivity and $\mathrm{X}$-axis is the wavelength in $\mathrm{nm}$.

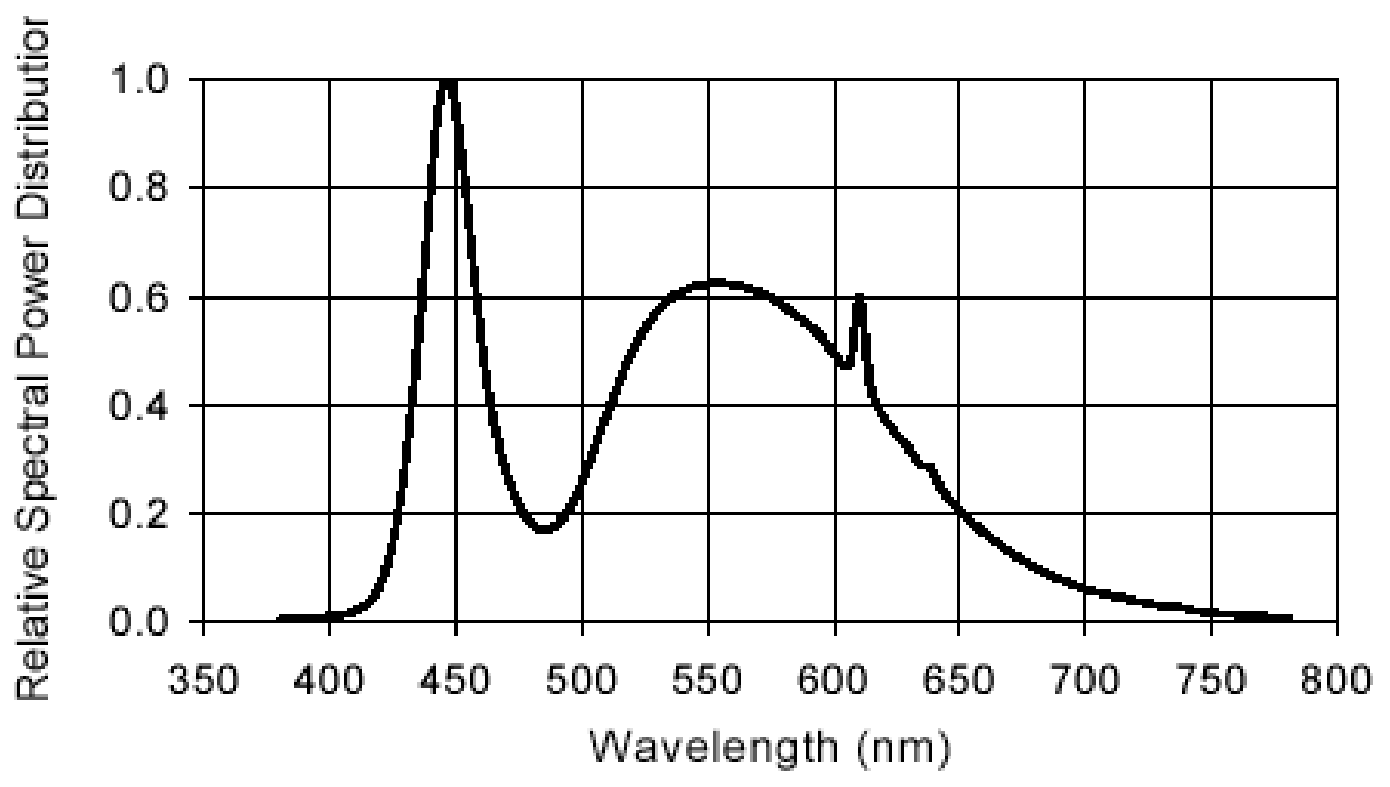

Figure 3: Optical Spectrum of a White Light LED 


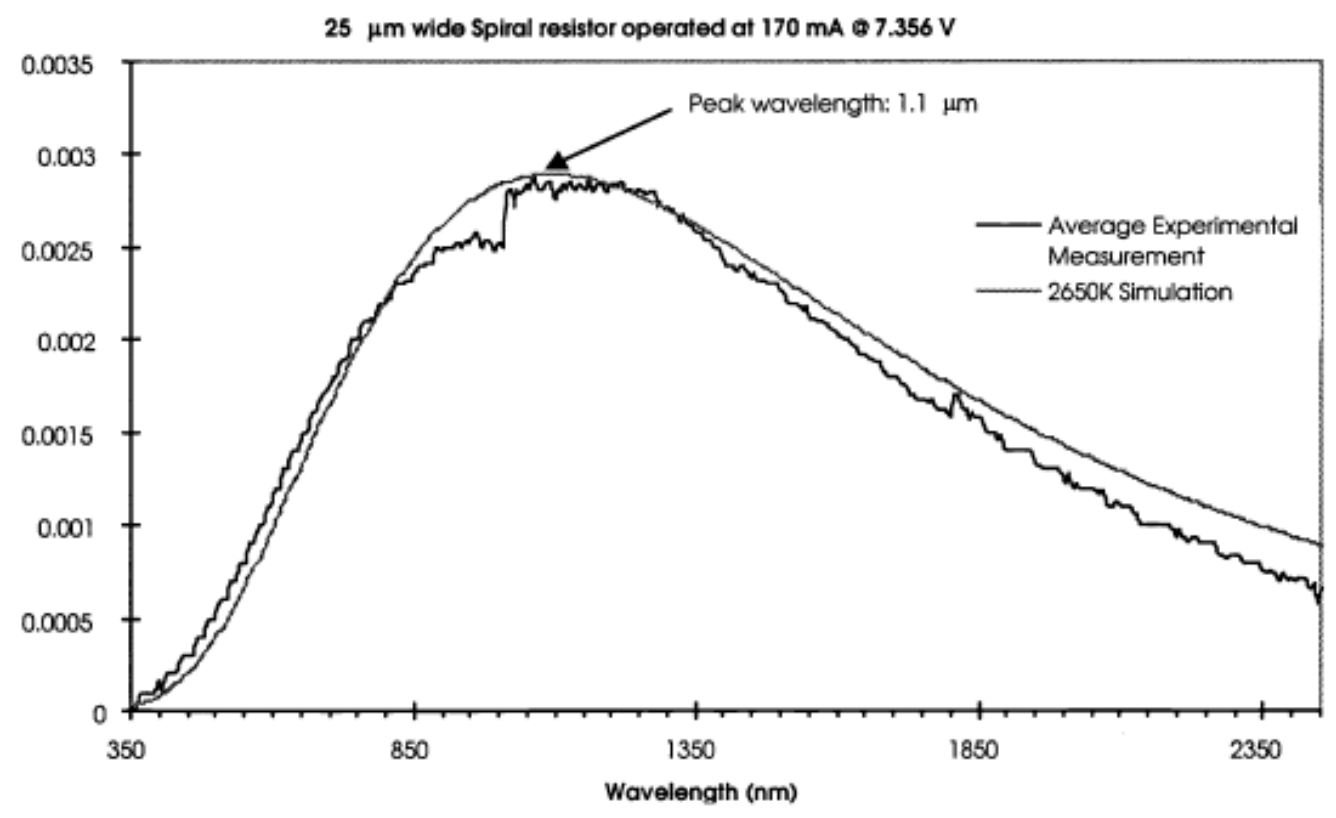

Figure 4: Optical Spectrum of a Tungsten Light Source.

\section{Experimental Measurements}

A variety of commercially available optical sources were evaluated as a suitable white light source. These are summarized in Table I. The Optek 372A diode is a narrow band source that is used in our relative measurement system. It was evaluated for comparison both in terms of power and coherence length.

Table I

\begin{tabular}{|l|l|l|l|}
\hline Source & Optical Power & Fiber Optical Power & $\begin{array}{l}\text { Optical Detector } \\
\text { Current }\end{array}$ \\
\hline $\begin{array}{l}\text { Control Development } \\
\text { HL-2000 }\end{array}$ & $30 \mathrm{~mW}$ & $\begin{array}{l}10 \mathrm{uW}(25 \mathrm{uW} \text { with } \\
\text { colimator })\end{array}$ & $2.5 \mathrm{nA}$ \\
\hline FTI-10 & NA & $300 \mathrm{nW}$ & $0.4 \mathrm{nA}$ \\
\hline $\begin{array}{l}\text { LumiLed White Light } \\
\text { LED }\end{array}$ & $3 \mathrm{~mW}$ & $1500 \mathrm{nW}$ & $0.2 \mathrm{nA}$ \\
\hline $\begin{array}{l}\text { Optek 372A 850nm } \\
\text { Narrowband LED }\end{array}$ & NA & $30 \mathrm{uW}$ & $70 \mathrm{nA}$ \\
\hline
\end{tabular}

The optical power was measured at the source, after coupling into the 50 micron core fiber, and finally at the optical detector after passing through the sensor and analyzer gaps. The source power numbers had to be measured with ND filters to avoid overload of the power meter. Also, all the light may not have been captured since it was not possible, due to the power head design, to get the power head any closer than about 1 " from the source. The measurement setup to evaluate optical coherence length is shown in Figure 5. The light couples out of the source and through a $2 \times 2$ coupler into the sensor. This part is just like the standard FTI-10 or Veloce approach to sensor readout. The reflected light was then analyzed using a second variable gap with the reflected light directed to a Silicon PIN detector. By scanning the variable gap, the peak coherence will be seen when 
the variable gap is equal to the sensor gap. The intensity of the interference will fall off according to the coherence length of the optical source. The variable gap can be implemented with a piezo-tuned mirror. It is intended to eventually use a piezo-tuned gap but there are critical requirements on both the flatness of the optical fiber and angle of the fiber cleave. This is particularly important for short coherence length sources. For these experiments the variable gap was implemented with a strain gauge mounted on a stressing bar. The commercial sensors have good control in terms of the flatness and angle between the two-mirrored surfaces of the sensor. The gap then is manually tuned by putting more or less stress on the bar. The time scale in the interference plots was primarily determined by how fast the strain gauge was stressed. It is also important that both the sensor and the variable gap have similar gaps in their quiescent state. For strain gauges this gap is about $15 \mathrm{um}$. For a temperature gauge at room temperature the gap is closer to 18 um.

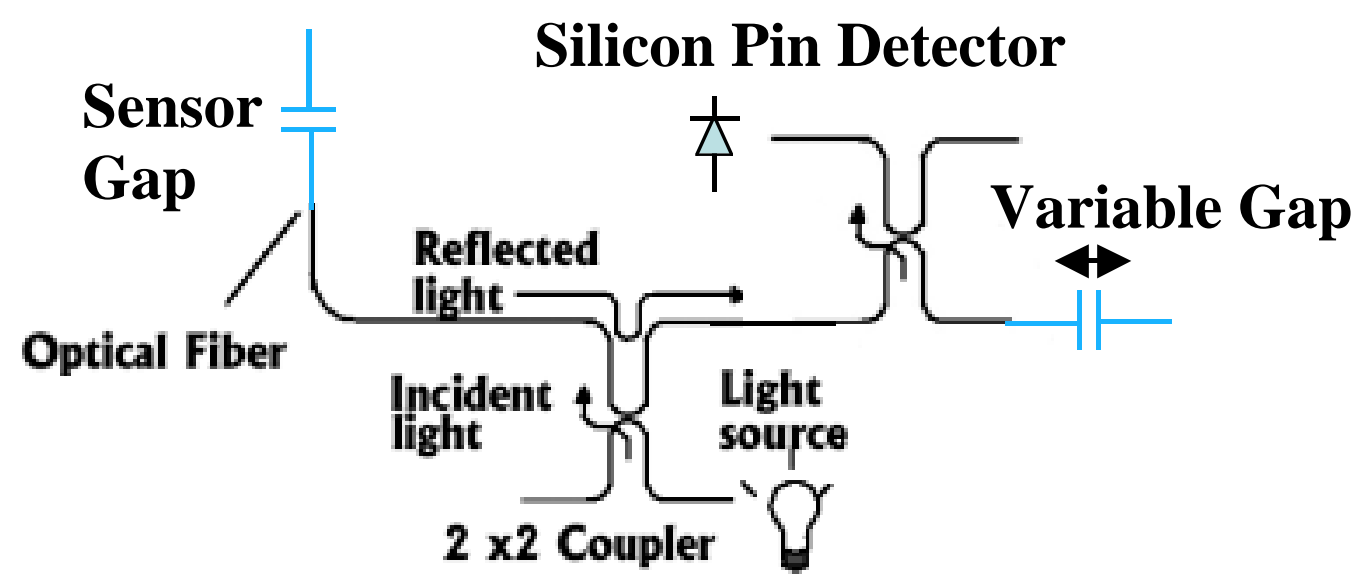

Figure 5: Optical Setup for coherence length measurements. 


\section{Results}

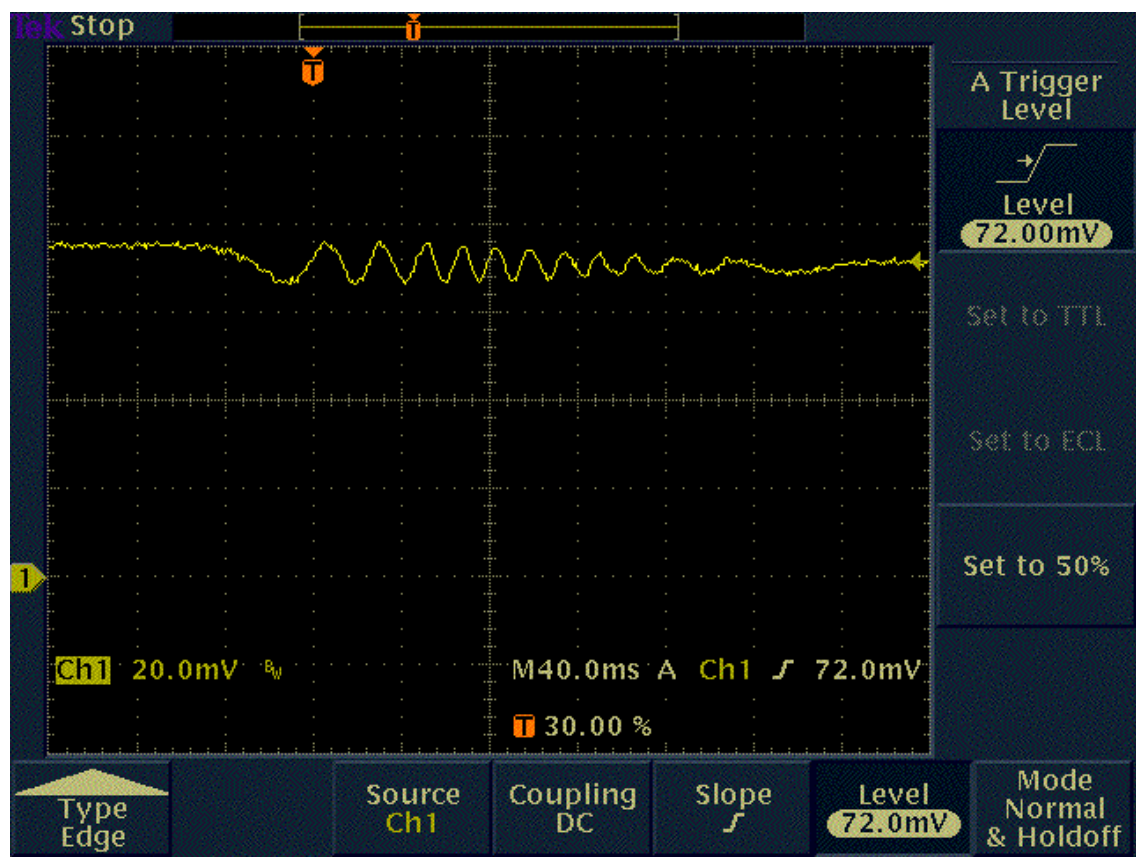

Figure 6: Interference plot for Optek 372A narrowband source

Figure 6 shows the type of interference from an $850 \mathrm{~nm}$ LED with a rather narrow optical spectrum. The resulting interference shows at least 11 peaks which indicates a relatively long coherence length which is useful for fringe counting systems such as the Veloce but not useful for an absolute readout system. This source also yielded the highest amplitude detector signal resulting in the best signal to noise of all the sources tested.

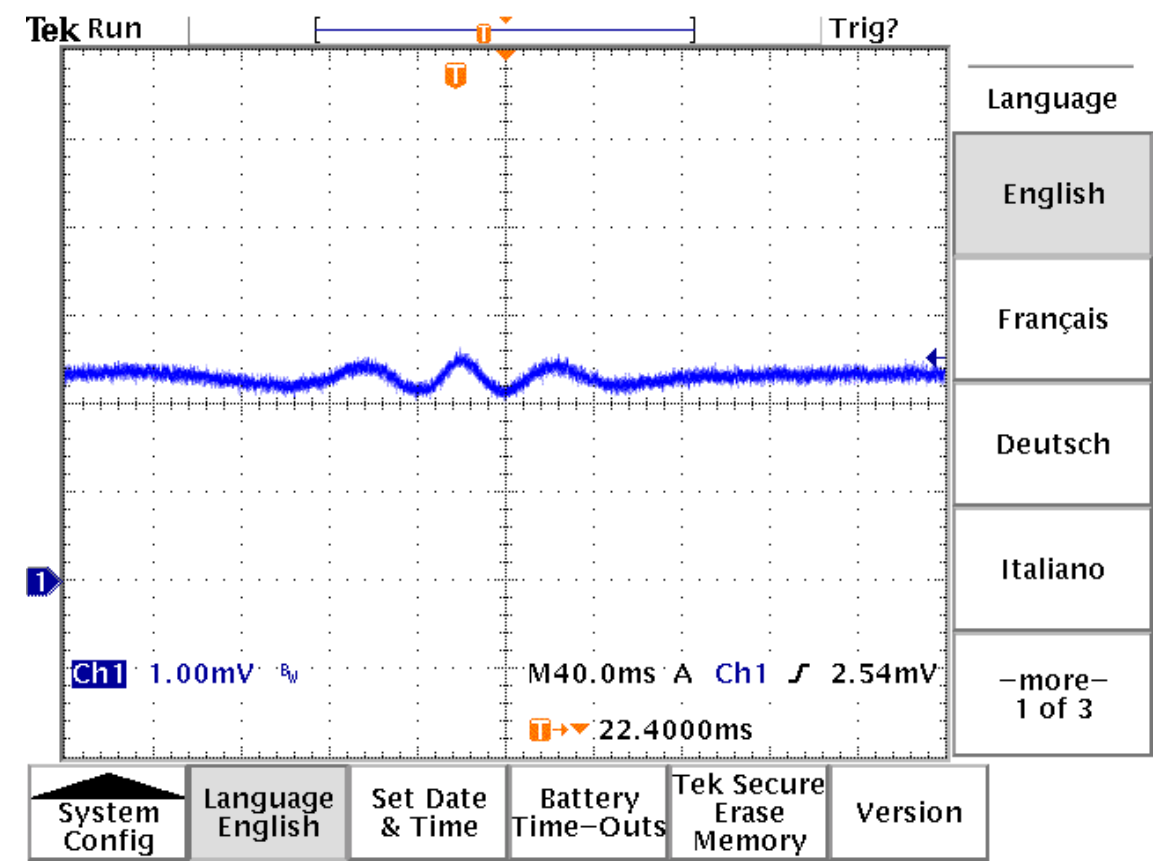

Figure 7: Interference plot for the Control Development White light source. 


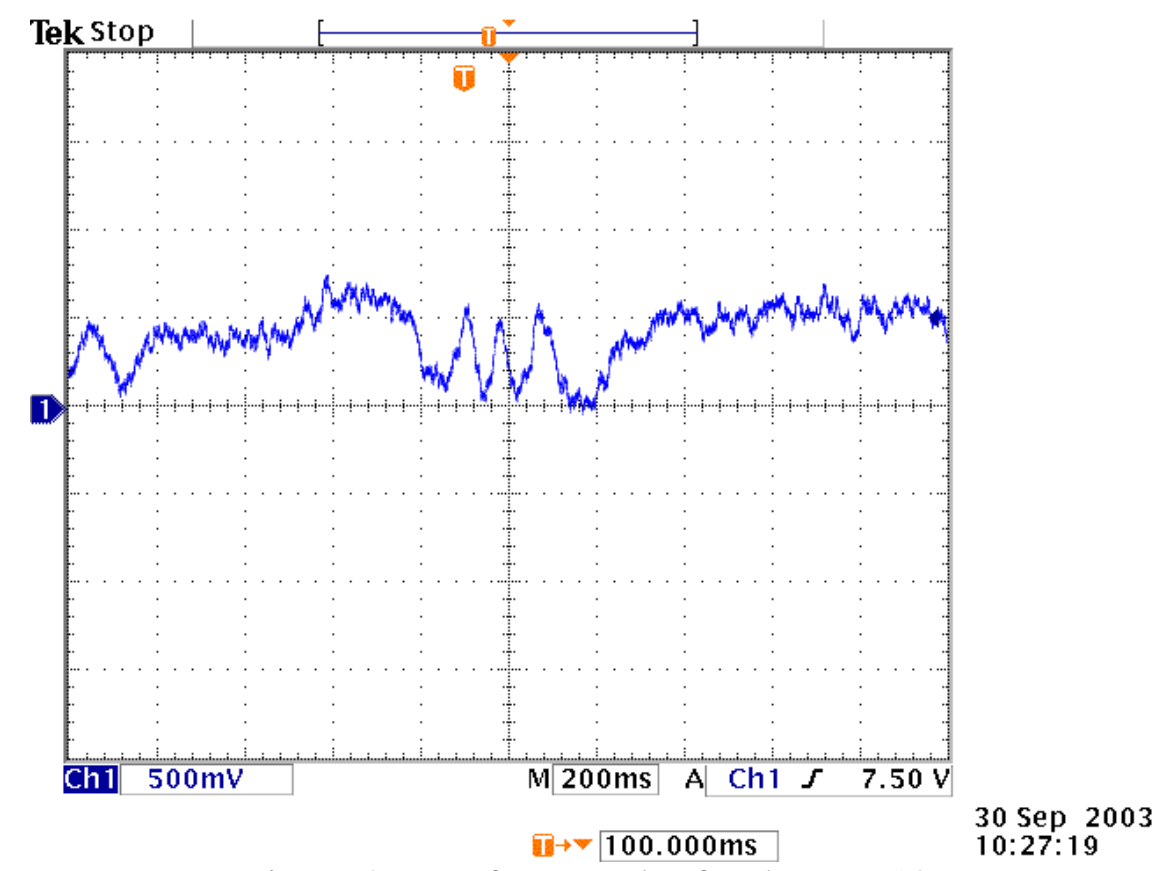

Figure 8: Interference plot for the FTI-10.

Figure 7 shows the interference plot for the Control Development white light source. In this case there are only about 3 peaks and it easy to localize the centroid of the interference plot. The signal level is near the minimum resolvable sensitivity on the scope which points to the need for higher amplitude sources. Figure 8 shows the interference plot for the FTI-10 light source. Because the signal level at the detector was in the noise for the scope, the light source was chopped with an optical chopper and the detector was fed to a lock-in amplifier in lieu of the scope. Figure 8 shows 3 peaks in the interference plot. Finally, in the case of the white light LED the signal was measured in a similar fashion with chopping and the lock-in detector. However, although a DC signal was measured on the lock-in indicating the presence of light, there was no interference peak. It is suspected that lack of the peak is from the lack of compatibility between the white light LED spectrum and the sensor reflectivity. (600 nm vs. $850 \mathrm{~nm}$ )

\section{Conclusions}

The light produced from a tungsten filament still appears to have the shortest coherence length for an absolute system. However, there are two main issues to achieving a higher power source:

1.) Better coupling from the source into the fiber. This coupling ratio currently is on the order of .001. Possibly with some optics design this number can be improved.

2.) Optical spectrum compatibility. The useful spectrum should be centered near 850 $\mathrm{nm}$ to be compatible with existing sensors. A great portion of the Tungsten spectrum is outside the wavelength range of interest and is either too short or too far into the IR. There is not an easy solution to this problem so hopefully the lost spectrum can be made up for to some extent by better coupling. 
The white light led is still an intriguing approach and could potentially overcome the problem of lost spectrum since its spectrum is determined by a phosphor emission spectrum that does not spread as much as the Tungsten source. However, existing phosphors are tailored as room light replacements and do not have any spectrum in the $850 \mathrm{~nm}$ range. It might be possible to build a sensor that works at $600 \mathrm{~nm}$. It might also be possible to find a phosphor that emits in the $850 \mathrm{~nm}$ range. In addition, there are white light LEDS that do not depend on phosphors and may emit further into the IR. In addition, work is progressing on a miniature Tungsten light source that may couple more efficiently to the optical fiber, so this may prove to be the source that meets our needs. 